\title{
Sizes of transition-region structures in coronal holes and in the quiet Sun
}

\author{
H. Tian ${ }^{1,2}$, E. Marsch ${ }^{1}$, C.-Y. Tu ${ }^{1,2}$, L.-D. Xia ${ }^{3}$, and J.-S. He ${ }^{2}$ \\ 1 Max-Planck-Institut für Sonnensystemforschung, Katlenburg-Lindau, Germany \\ e-mail: tianhui924@163.com; marsch@mps.mpg.de \\ 2 Department of Geophysics, Peking University, Beijing, PR China \\ e-mail: chuanyitu@pku.edu.cn \\ 3 Department of Space Science and Applied Physics, Shandong Univ. at Weihai, Weihai, Shandong, PR China \\ e-mail: xld@ustc.edu.cn
}

Received 11 December 2007 / Accepted 14 February 2008

\begin{abstract}
Aims. We study the height variations of the sizes of chromospheric and transition-region features in a small coronal hole and the adjacent quiet Sun, considering images of the intensity, Doppler shift, and non-thermal motion of ultraviolet emission lines as measured by SUMER (Solar Ultraviolet Measurements by Emitted Radiation), together with the magnetic field as obtained by extrapolation from photospheric magnetograms.

Methods. In order to estimate the characteristic sizes of the different features present in the chromosphere and transition region, we have calculated the autocorrelation function for the images as well as the corresponding extrapolated magnetic field at different heights. The Half Width at Half Maximum (HWHM) of the autocorrelation function is considered to be the characteristic size of the feature shown in the corresponding image.

Results. Our results indicate that, in both the coronal hole and quiet Sun, the HWHM of the intensity image is larger than that of the images of Doppler-shift and non-thermal width at any given altitude. The HWHM of the intensity image is smaller in the chromosphere than in the transition region, where the sizes of intensity features of lines at different temperatures are almost the same. But in the upper part of the transition region, the intensity size increases more strongly with temperature in the coronal hole than in the quiet Sun. We also studied the height variations of the HWHM of the magnetic field magnitude $B$ and its component $\left|B_{z}\right|$, and found they are equal to each other at a certain height below $40 \mathrm{Mm}$ in the coronal hole. The height variations of the HWHM of $\left|B_{z} / B\right|$ seem to be consistent with the temperature variations of the intensity size.

Conclusions. Our results suggest that coronal loops are much lower, and magnetic structures expand through the upper transition region and lower corona much more strongly with height in the coronal hole than in the quiet Sun.
\end{abstract}

Key words. Sun: corona - Sun: transition region - Sun: UV radiation - Sun: magnetic fields

\section{Introduction}

The solar transition region, where above the photosphere the temperature increases rapidly and the density drops dramatically, is a rather inhomogeneous and dynamic layer between chromosphere and corona (for a review see Mariska 1992). The transition region is believed to play an important role in the origin of the solar wind and in coronal heating (Hassler et al. 1999; Tu et al. 2005a; Chae et al. 1998; Hansteen 1993).

One of the most prominent features in the chromsphere and transition region is the magnetic network (Reeves 1976), which is the upward extension of the supergranular boundaries above the photosphere. The network manifests itself as bright lanes on the radiance images of emission lines, and is characterized by clusters of magnetic flux concentrations (Patsourakos et al. 1999). The typical network cell has a size of 20000 to $30000 \mathrm{~km}$ (Simon \& Leighton 1964), and lasts for about 20 to $50 \mathrm{~h}$ (Simon \& Leighton 1964; Schrijver 1997; Raju et al. 1998). It is suggested that part of the network flux opens into the corona and has a funnel shape, while the rest of the network consists of a dense population of low-lying loops with lengths less than $10^{4} \mathrm{~km}$ (Dowdy et al. 1986) and varying orientations. A funnel may be connected to the solar wind or just be the foot of a large coronal loop (Peter 2001).

Another prominent characteristic of the transition region is the observed red shift of most transition region lines (see the reviews by Mariska 1992 and Wilhelm et al. 2007). However, a net blue shift of the upper transition region line Ne VIII ( $\lambda 770)$ has been observed by SUMER (Solar Ultraviolet Measurements by Emitted Radiation) (Dammasch et al. 1999). The transition from redshift to blueshift occurs at an electron temperature of about $5 \times 10^{5} \mathrm{~K}$; the detailed temperature variation of the Doppler shift can be found in Peter \& Judge (1999), Teriaca et al. (1999), and Xia et al. (2004).

Besides line intensity and Doppler shift, spectroscopic diagnostics can also provide us with information on non-thermal motions of the emitting ions. The observed non-thermal broadenings could correspond to small-scale laminar flows, waves, or turbulence, each of which may play a role in energy transport and coronal heating (Chae et al. 1998). The non-thermal velocity at temperatures below $2 \times 10^{4} \mathrm{~K}$ is less than $10 \mathrm{~km} \mathrm{~s}^{-1}$. On average, it increases with temperature, reaches a peak value of $30 \mathrm{~km} \mathrm{~s}^{-1}$ around $3 \times 10^{5} \mathrm{~K}$, and then decreases with temperature (Chae et al. 1998). The average non-thermal velocities of 
transition region lines are found to be larger in coronal holes than in quiet Sun regions (Lemaire et al. 1999).

Investigating the temperature or height dependence of a specific line feature such as radiance, Doppler shift and non-thermal width in the solar atmosphere is very helpful in understanding the structure of the solar atmosphere and the relevant physical processes (Chae et al. 1998). Based on a two-dimensional autocorrelation method, Patsourakos et al. (1999) analyzed six intensity images of transition region lines with different formation temperatures, and thus studied the temperature variation of the network-boundary thickness indicated by the HWHM of the autocorrelation function (ACF) in a quiet Sun region. They concluded that network boundaries have an almost constant size up to a temperature of about $10^{5.4} \mathrm{~K}$ and then fan out rapidly at coronal temperatures. Gontikakis et al. (2003) extended this work by studying the sizes of structures in Doppler shift and width, but only for three lines in a quiet Sun region. They found that the size of bright radiance features is larger than that of the structures of the dopplergram and Doppler width. They also found that the sizes of C IV line are smaller than those of Si II. Ravindra \& Venkatakrishnan (2003) used the autocorrelation technique, as well as the so-called structure function, to study the life time and length scale of the network cells seen in images of He II (304 $\AA$ ) passband observed by the Extreme-ultraviolet Imaging Telescope (EIT). The two methods were also applied to the extrapolated magnetic field at different heights in their paper.

Here we will analyse three different data sets obtained by SUMER on the Solar and Heliospheric Observatory (SOHO) and report the new results on the sizes of chromospheric and transition-region features. The autocorrelation technique was applied to images of intensity, Doppler shift, and non-thermal width of several lines, as well as the corresponding extrapolated magnetogram at different heights. The HWHM of the autocorrelation function is considered to be the characteristic size of the feature in the corresponding image. We will investigate the height variations of the sizes of different features, compare the differences found between coronal hole and quiet Sun, and subsequently discuss the relevant physical implications.

\section{Observations and data reduction}

Three data sets obtained by SUMER were included in this study. The first one was taken from 18:00 on March 7 to 17:42 UTC of the next day in 1997. During this period, SUMER observed an equatorial coronal hole and the surrounding quiet Sun. More information about this observation can be found in Lemaire et al. (1999), Aiouaz et al. (2005) and Aiouaz (2008), in which this data set was also used. We selected 6 emission lines including Ne VIII (770.428 ̊), O V (760.43 $⿱$ ), O IV (787.70 ̊), S V (786.47 $\AA$ ), N IV (765.15 $\AA$ ) and N III (764.36 $\AA$ ) for our study. We averaged the data over 4 exposures, which greatly improved the signal-to-noise level and produced a new data set with a similar pixel size in the direction of the solar $X$ and $Y$ coordinate.

The other two data sets were obtained on September 28, 1996 from 15:10 to 03:18 UTC on the next day in an equatorial region of the quiet Sun, and on 16 April from 8:21 to $15: 48$ UTC in a south polar region. In each of these data sets, more than 40 EUV lines with a wide temperature coverage were included in the spectral windows to observe almost the same region on the solar disk. However, only part of them turned out to be strong and clean enough for our study (16 lines in the quiet Sun and 13 lines in the coronal hole). The selected lines for the quiet Sun region are Si I (1258.77 $\AA)$, O I (948.7 $\AA$ ),

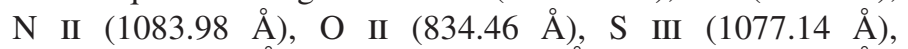
Si III (1113.24 $\AA$ ), Si IV (1393.74 $\AA$ ), N III (991.57 $\AA$ ), S IV $(1072.97 \AA), \quad S$ VI $(933.41 \AA), \quad O \quad$ V $(629.74 \AA ̊ \AA)$, O VI (1037.63 $\AA)$, Fe VIII (1062.44 $\AA$ ), Ne VII (562.32 $\AA$ ), Ne VIII (770.428 $\AA$ ), and Mg X (624.97 $)$. The chosen lines for the coronal hole region are Si I (1258.77 $\AA$ ), C II (1037.03 $\AA$ ), O II (834.46 ̊), S III (1077.14 ̊), Si III (1113.24 Si IV (1393.74 $\AA), \quad N$ III $(991.57 \AA), S$ IV (1072.97 $\AA)$, S VI (933.41 $\AA$ ), O VI (1037.63 $\AA$ ), Fe VIII (1062.44 $\AA$ ), Ne VII (562.32 $\AA$ ), and Ne VIII (770.428 $\AA$ ). We took the values of the wavelengths from Curdt et al. (2004). The formation temperatures were taken from Xia (2003) and the Chianti data base (Dere et al. 1997; Landi et al. 2006) and can be found in Figs. 3 and 4. For each of these two data sets, the observed regions are not exactly the same for different lines, especially in the quiet Sun region for which the spatial shift between two images can reach half of the size of the observation region.

The standard SUMER procedures for correcting and calibrating the data were applied, including local-gain correction, flat-field correction, geometrical-distortion and dead-time correction. A single Gaussian fit was applied to each observed spectral line profile. By integrating over a fitted profile, we derived the total count rate of the line and thus obtained its intensity that was used to build an image of each line. Figure 1 shows the intensity images of N IV (left) and Ne VIII (right) for the first data set. The two sub-regions enclosed by the white rectangles are defined as the coronal-hole and quiet Sun region, respectively, which are further studied in detail. Figure 2 shows the intensity images of S IV (left) and Ne VIII (right) of the second data set (upper frames) and of the third data set (in the lower frames).

Since for the second and third data set the exposure times are only $30 \mathrm{~s}$ and $20 \mathrm{~s}$, respectively, the Doppler shift and width produced by a single Gaussian fit could not be reliably determined for many of the spectra. So for these two data sets, we only studied the characteristic sizes as inferred from intensity images. But for the first data set, since the exposure time was $90 \mathrm{~s}$, and as we binned the data over four exposures, we could determine the Doppler shift and non-thermal width of any line very well.

To determine the Doppler shift, we first estimated the instrumental shift caused by thermal deformations of the optical system of SUMER by using the same technique adopted in Dammasch et al. (1999). Since there is no chromospheric line in our spectral windows, we could not carry out an absolute wavelength calibration by using a certain cold line. Instead, we followed the method employed by Aiouaz et al. (2005) and obtained the dopplergram after assuming a mean Doppler shift value for all the spectra of each line in the first data set. We took a value of $-2.5 \mathrm{~km} \mathrm{~s}^{-1}$ (blue shift) for Ne VIII and $7 \mathrm{~km} \mathrm{~s}^{-1}$ (red shift) for the other lines from Xia (2003), in which the results were based on a statistical study.

The measured broadening of a line profile results from a combination of instrumental broadening, thermal and nonthermal motion (Chae et al. 1998; Xia 2003). For the line profiles recorded by SUMER, instrumental broadening can be removed

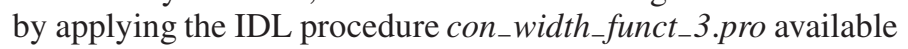
in $S S W$ (the Solar Software). With the assumption of local thermal equilibrium the corresponding thermal velocity, and thus its temperature, can be calculated for a certain ion. After removing the instrumental and temperature broadening, we thus obtained the image of the non-thermal velocity for each line in the first data set. 

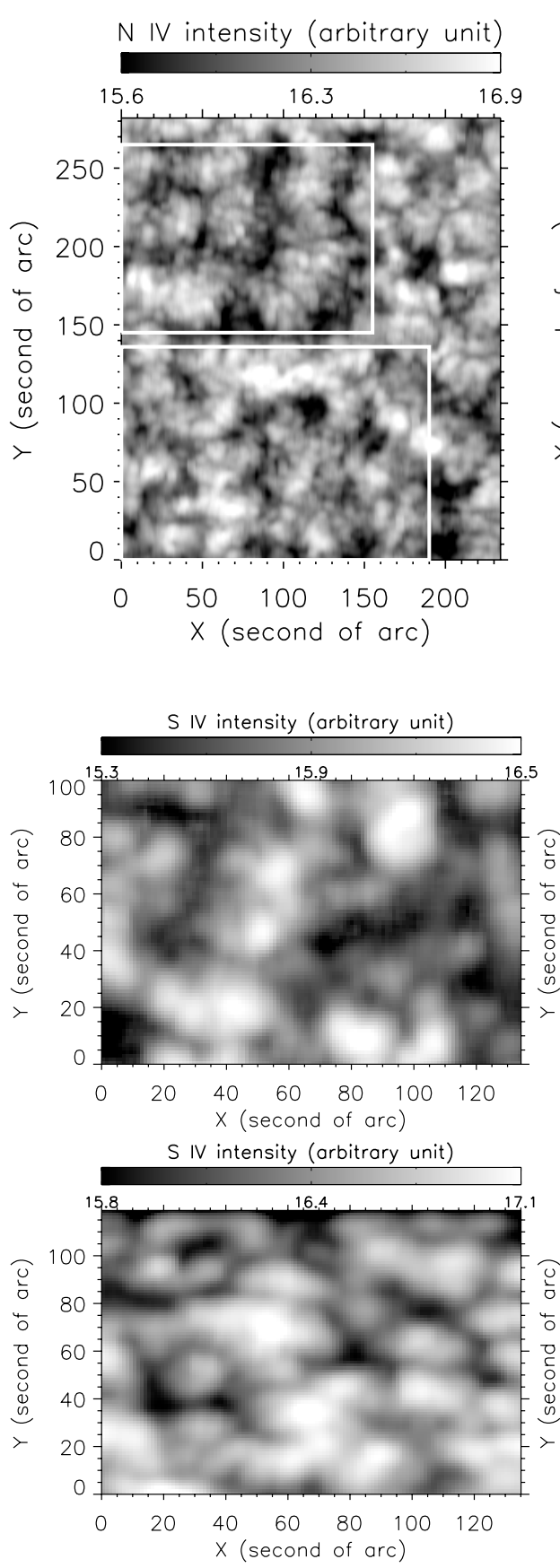
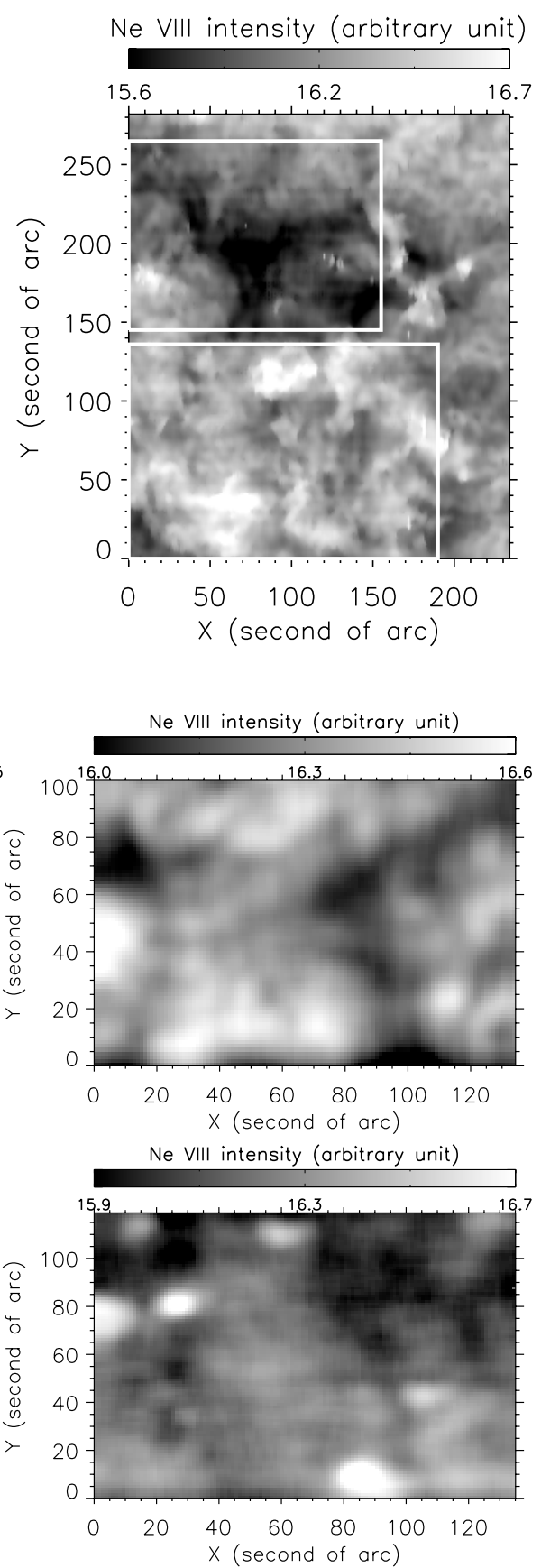

Fig. 1. Intensity images of N IV (left) and Ne VIII (right) in the first data set (from 18:00 on March 7 to $17: 42$ on March 8 in 1997). The intensity images are in logarithmic scale. The two sub-regions enclosed by white lines are defined as the coronal-hole and quiet-Sun parts and studied in detail.
Fig. 2. Intensity images of S IV (left) and $\mathrm{Ne}$ VIII (right) of the second data set (upper panel, observed from 15:10 on September 28 to $03: 18$ on September 29) and the third data set (lower panel, observed from 8:21 to 15:48 on April 16). The intensity images are in logarithmic scale.
In order to investigate the links between the 3-D magnetic structures and the long-lasting transition-region features, we successfully reconstructed the magnetic structure above the photosphere by using the force-free model proposed by Seehafer (1978), following the work of many authors (Marsch et al. 2004; Wiegelmann et al. 2005; Tu et al. 2005a,b; Marsch et al. 2006; He et al. 2007; Tian et al. 2007, 2008). Here we reconstructed a potential 3-D magnetic field, by applying the same method and using the observed magnetograms which correspond to the area observed by SUMER in each data set. For the first data set, a Michelson Doppler Imager (MDI) magnetogram was chosen in the same way as in Aiouaz et al. (2005). For the other two data sets, we first obtained a full-disk Kitt-Peak magnetogram observed during the scan period of SUMER, and then used a sub-magnetogram with a size $40^{\prime \prime}$ larger than the SUMER observation region to perform the field extrapolation. The line-of-sight magnetic field components measured by the magnetographs were then converted to the component perpendicular to the solar surface, by simply considering and correcting for the cosine effect. We thus calculated the magnetic field up to $40 \mathrm{Mm}$ above the photosphere.

\section{Results and discussion}

The main motivation for this work is to study the temperature or height variation of the sizes of transition region structures in a coronal hole and the quiet Sun. In order to estimate the characteristic sizes of different features present in the chromosphere, transition region and lower corona, we calculated the ACF for each image of intensity, Doppler shift, and non-thermal width of EUV lines obtained by SUMER, as well as the corresponding extrapolated magnetic field at different heights. The calculation of ACF is based on Fourier analysis and can provide a statistical estimate of the scale length of any features in an image. We first 


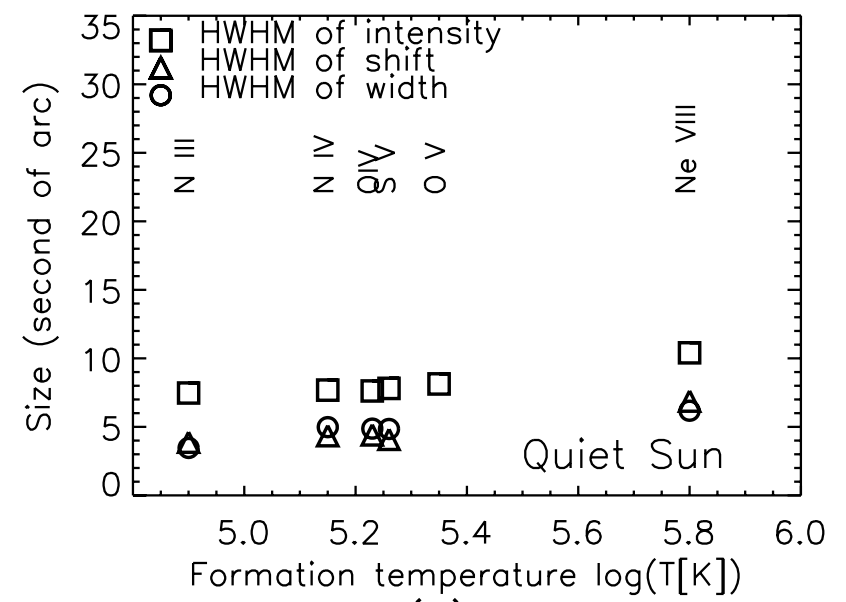

(a)

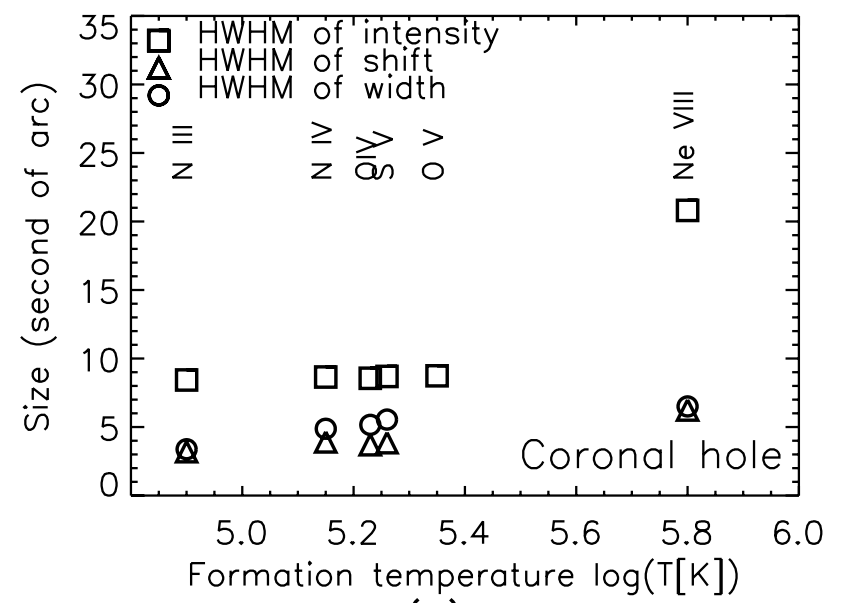

(c)

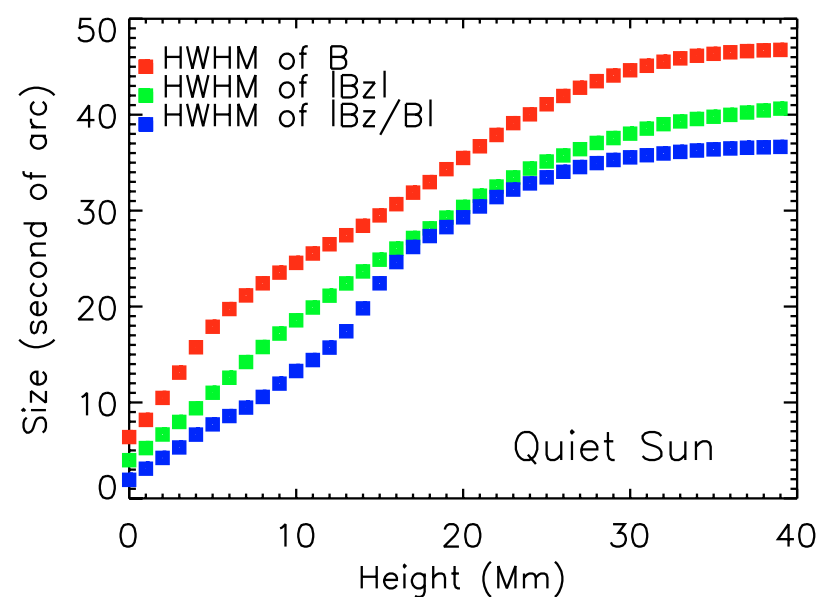

(b)

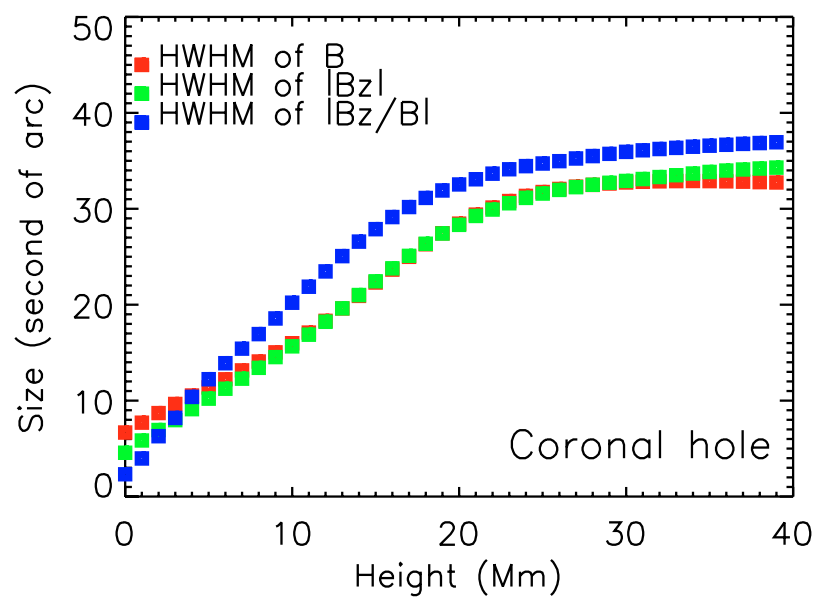

(d)

Fig. 3. Temperature/height variations of the characteristic sizes of intensity, Doppler shift and non-thermal width (left), as well as of the extrapolated magnetic field (right) in the quiet Sun (upper panel) and coronal hole (lower panel) for the first data set.

calculated the 2-D ACF of each image by shifting it in both dimensions, and then took its angular average in rings of integer radius and derived a curve of the correlation coefficient against radial distance (Patsourakos et al. 1999). The HWHM of the ACF is considered to be the scale size of the feature, while the distance between the primary and secondary peak corresponds to the mean distance between two adjacent features (Patsourakos et al. 1999; Gontikakis et al. 2003; Ravindra \& Venkatakrishnan 2003). Here we are only interested in the HWHM. The smaller the features, the sharper the central peak of the ACF will be (Gontikakis et al. 2003).

In Fig. 3a and c, the inferred HWHM of intensity, Doppler shift and non-thermal width are shown for all the lines selected in the first data set for the quiet Sun and coronal hole respectively. The O V (760.43 $\AA$ ) line is blended with O V (760.21 $\AA$ ) (Aiouaz 2008). The blend is weak and the line is about 5 pixels away from O V (760.43 $\AA$ ), so that we can still study the intensity image. But the blend can have a large impact on the determination of Doppler shift and non-thermal width. Therefore, we did not study the images of the shift and width of this line. Figure 3 demonstrates that the intensity size is larger than that associated with the Doppler shift and non-thermal width, a result has been found before by Gontikakis et al. (2003). It could be due to the orientation of the magnetic field, since the observed Doppler shift and non-thermal motion correspond to the line-of-sight components, which are expected to be large only in the vertical legs of magnetic loops. We found that the sizes of all three features are rather stable in the middle transition region. The sizes of the Ne VIII structures are larger than those derived by means of middle-transition-region lines $(\log T<5.7)$, which is especially true in the coronal hole for the size of Ne VIII intensity structures. The strong increase of the size of the Ne VIII intensity features indicates that the magnetic field expands much more strongly with height in coronal hole than in the quiet Sun region. The field of view selected to define the coronal hole in this data set includes a relatively bright structure in the intensity image of Ne VIII, centered at $(x, y)=\left(20^{\prime \prime}, 170^{\prime \prime}\right)$ according to the axes in Fig. 1. This bright feature was omitted from the delimitation of the coronal hole in Lemaire et al. (1999) and Aiouaz et al. (2005). We computed the HWHM after omitting this structure (the field of view selected to define the coronal hole was changed from $\left(x: 0^{\prime \prime}-155^{\prime \prime} ; y: 145^{\prime \prime}-265^{\prime \prime}\right)$ into $\left.\left(x: 50^{\prime \prime}-155^{\prime \prime} ; y: 145^{\prime \prime}-265^{\prime \prime}\right)\right)$. The result reveals that except for the size of Ne VIII intensity feature, which changes from about $20^{\prime \prime}$ to $18^{\prime \prime}$ and does not influence our result, all of the sizes of features do not show visible changes. Thus, we believe that the influence of the bright feature on our results can be neglected.

The two right panels of Fig. 3 show the height variation of the sizes of the corresponding (extrapolated) coronal field parameters $B,\left|B_{z}\right|$ and $\left|B_{z} / B\right|$ in the quiet Sun and coronal hole, 


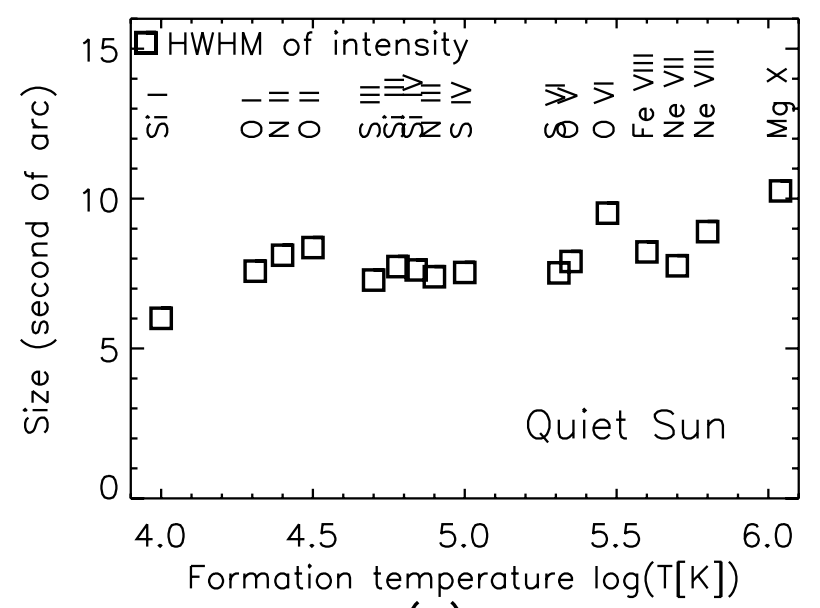

(a)

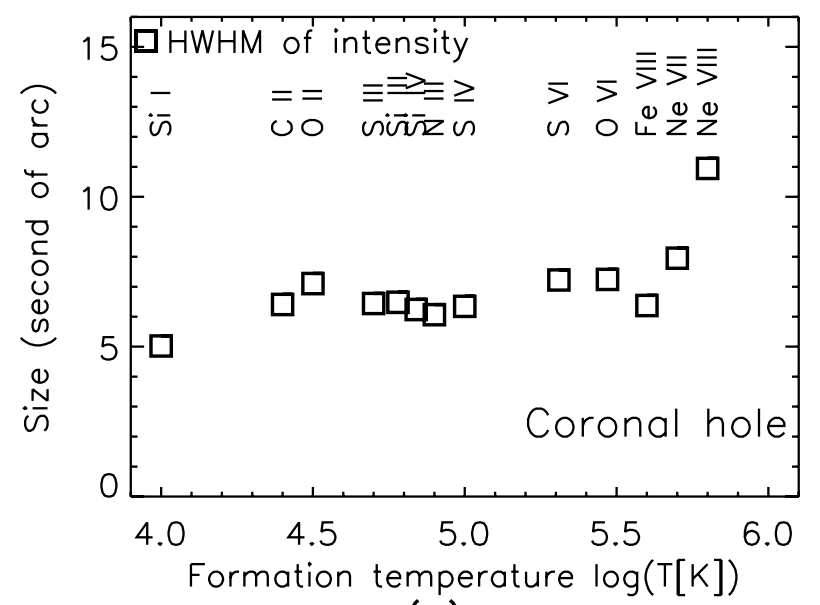

(c)

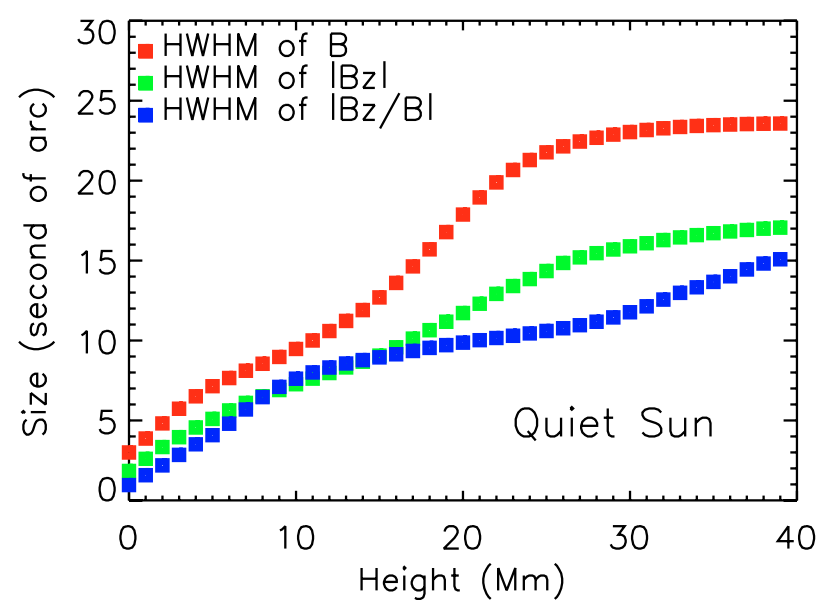

(b)

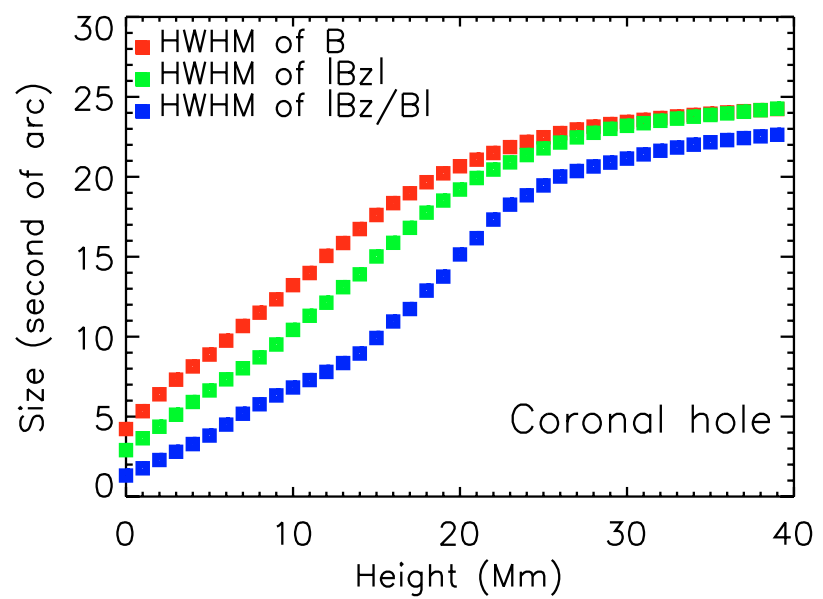

(d)

Fig. 4. Temperature/height variations of the characteristic sizes of line intensity structures (left) and of structures inferred from the coronal magnetic field parameters (right) in the quiet Sun (upper panel, for the second data set) and coronal hole (lower panel, for the third data set).

respectively. In the coronal hole, the HWHM of $\left|B_{z}\right|$ and $B$ increase linearly with height below $10 \mathrm{Mm}$. The sizes (in terms of HWHM) of the two features are equal to each other at $10 \mathrm{Mm}$, implying that small loops only reside below $10 \mathrm{Mm}$ and the magnetic field is entirely open above $10 \mathrm{Mm}$. This result is consistent with the result of Wiegelmann \& Solanki (2004), in which high and long closed loops are found to be extremely rare in coronal holes. In contrast, in the quiet Sun region the HWHM of $\left|B_{z}\right|$ is always smaller than that of $B$, which means that loops with different heights up to $40 \mathrm{Mm}$ exist in the quiet Sun region. $\left|B_{z} / B\right|$ is an indicator of the inclination of a magnetic field line with respect to the horizontal direction. So the height variation of the size of $\left|B_{z} / B\right|$ can reveal the expansion of funnels. It is apparent that below $10 \mathrm{Mm}$ the size of $\left|B_{z} / B\right|$ increases much more strongly with height in the coronal hole than in the quiet Sun, indicating that funnels expand more strongly with height in the coronal hole. This is consistent with the result that the size of the Ne VIII intensity structures increases dramatically in the coronal hole. We found that at $10 \mathrm{Mm}$, the HWHM of $\left|B_{z} / B\right|$ is about $10^{\prime \prime}$ in the quiet Sun and 20" in the coronal hole, which is almost the same as the HWHM of the Ne VIII intensity features in both regions.

The results obtained from the second (quiet Sun) and third (coronal hole) data set are presented in Fig. 4. The formation temperatures of the lines selected here range from $10^{4} \mathrm{~K}$ to
$10^{6.02} \mathrm{~K}$, and so we can study the variation of the size of features in the intensity image from the chromosphere to the lower corona, which is shown on the two left panels of the figure. If we consider the HWHM of bright radiance features to be the size of the network (Patsourakos et al. 1999), then it is clear that the network size increases from the chromosphere to the transition region within the temperature range of $\log T=4.0-4.4$ in both the quiet Sun and the coronal hole. In the transition region $(\log T=4.4-5.7)$, we found that the network size almost does not change. Beyond $\log T=5.7$, we can see an increase with temperature in network size, more dramatic in the coronal hole than the quiet Sun region, which is consistent with the result of the first data set. The Mg X line in the second data set is blended with three other lines, which contribute about $20 \%$ to the total intensity (Teriaca et al. 2002) and should not have a large influence on the results. Gontikakis et al. (2003) found that the feature sizes inferred from the middle transition region line (C IV, $\log T=5.0$ ) are smaller than those from the chromospheric line (Si II, $\log T=4.4$ ). However, such a relation is not obvious in Fig. 4.

As for the results obtained from the second and third data set, concerning the properties of the coronal magnetic field, the height variations of the sizes (derived from the HWHM) of features in $\left|B_{z}\right|$ and $B$ generally are similar to those found for the first data set. These results can be seen in the two right 
panels of Fig. 4. One difference is the height above which the two sizes become equal in the coronal hole, which occurs at about $25 \mathrm{Mm}$ and implies that small loops reside only below $25 \mathrm{Mm}$ in this coronal hole. The increment of the HWHM for $\left|B_{z} / B\right|$ with height is almost the same below $10 \mathrm{Mm}$ in both areas, implying that the magnetic structures are of similar size in the quiet Sun and coronal hole at low heights above the photosphere. It is clear that from about $10 \mathrm{Mm}$ to $20 \mathrm{Mm}$, the size of $\left|B_{z} / B\right|$ increases much more strongly with height in the coronal hole, a trend perhaps indicating that funnels expand with height in the lower corona more strongly in the coronal hole than in the quiet Sun. This conclusion is consistent with the finding that the size of the Ne VIII intensity features dramatically increases with temperature in the coronal hole. It is interesting to note that in both regions, the change in the HWHM of $\left|B_{z} / B\right|$ from $10 \mathrm{Mm}$ to $20 \mathrm{Mm}$ seems to be consistent with the change in the HWHM of the intensity with temperature from the middle transition region to the lower corona.

Inspection of the two left panels of Fig. 4 shows that the sizes of intensity structures as seen in the different lines originating in the middle transition region are not exactly the same, which may reflect that the observation areas for the different lines are not exactly the same in the second and third data set. Another possible reason may be that the images, to which we applied the autocorrelation technique, were not large enough in size, and thus they perhaps do not represent an ideal sample in the statistical sense. However, we calculated the ACF for artificial images having different sizes but the same scale of the features, and found that the HWHM of the ACF for an image does not depend largely on its overall size. Thus, the overall sizes of the images used in this paper are large enough for our study. On the other hand, we are mainly interested in the height variation with size, but not so much in the absolute value of the scale of a feature. Therefore, we believe that our results are reliable for that purpose.

One may find that the linear increase of the HWHM of $\left|B_{z}\right|$ or $B$ as a function of altitude, up to $10 \mathrm{Mm}$, is not followed by the network structures measured at temperatures lower than the Ne VIII formation temperature, as shown in Figs. 2 and 4. Especially from $\log T=4.4$ to $\log T=5.7$, the network size almost does not change. This result seems to indicate that in the transition region, plasmas with different temperatures can coexist at the same height (Marsch et al. 2006), and the structures of their emission sources have comparable sizes. Another possibility could be that the emission of the solar plasma in the lower transition region is intermittent and shows substantial temporal and spatial variability, which could influence the computed HWHM (Wilhelm et al. 1998; Brković et al. 2003; Gontikakis et al. 2003).

\section{Summary and conclusion}

For the scaling analysis, we applied the autocorrelation technique to the images of intensity, Doppler shift, and non-thermal width of several EUV lines measured by SUMER, as well as to the corresponding magnetic field maps obtained by extrapolation of photospheric magnetograms to different heights. The HWHM of the ACF is considered to determine the characteristic size of a feature in the respective image.

Concerning the sizes of magnetic structures in the transition region, a different variation with height was found for the sizes (in terms of HWHM) of features in the extrapolated field magnitude $B$ and its component $\left|B_{z}\right|$. The sizes of the two features are equal at a certain height in the coronal hole. While in the quiet Sun region, the HWHM of $\left|B_{z}\right|$ is smaller than that of $B$ at any height below $40 \mathrm{Mm}$. This indicates that loops are much lower in the coronal hole than in the quiet Sun region. The height variation of the HWHM of $\left|B_{z} / B\right|$ suggests that an open magnetic structure expands through the upper transition region and lower corona more strongly in coronal holes than in the quiet Sun. Furthermore, we found that in the upper part of the transition region, the size of a line-intensity feature increases with temperature more strongly in the coronal hole than in the quiet Sun. We also corroborated a result found previously that the features in dopplergrams and images of the non-thermal width are smaller than those in the intensity images, a finding that may be due to the line-of-sight effect.

Acknowledgements. We thank Dr. W. Curdt, Dr. M. S. Madjarska, Dr. L. Teriaca, and Dr. D. Innes for their help in SUMER data analysis. We also thank the referee Dr. Costis Gontikakis for his careful reading of the paper and for the comments and suggestions.

H. Tian, C.-Y. Tu, and J.-S. He are supported under contracts 40574078, 40336053, 40436015, and by the Beijing Education Project XK100010404, as well as the foundation Major Project of National Basic Research contract 2006CB806305. H. Tian is also supported by China Scholarship Council for his stay in the Max-Planck-Institut für Sonnensystemforschung in Germany.

The SUMER project is financially supported by DLR, CNES, NASA, and the ESA PRODEX programme (Swiss contribution). SUMER and MDI are instruments on board SOHO, an ESA and NASA mission. We thank the teams of SUMER and MDI for the spectroscopic and magnetic field data. We also thank the NSO/Kitt Peak observatory for the use of the magnetic field data.

\section{References}

Aiouaz, T. 2008, ApJ, 674, 1144

Aiouaz, T., Peter, H., \& Lemaire P. 2005, A\&A, 435, 713

Brković, A., Peter, H., \& Solanki, S. K. 2003, A\&A, 403, 725

Chae, J., Schühle, U., \& Lemaire, P. 1998, ApJ, 505, 957

Curdt, W., Landi, E., \& Feldman, U. 2004, A\&A, 427, 1045

Dammasch, I. E., Wilhelm, K., Curdt, W., \& Hassler, D. M. 1999, A\&A, 346, 285

Dere, K. P., Landi, E., Mason, H. E., Monsignori-Fossi, B. F., \& Young, P. R. 1997, A\&AS, 125, 149

Dowdy, J. F. Jr., Rabin, D., \& Moore, R. L. 1986, Sol. Phys., 105, 35

Gontikakis, C., Peter, H., \& Dara, H. C. 2003, A\&A, 408, 743

Hassler, D. M., Dammasch, I. E., Lemaire, P., et al. 1999, Science, 283, 810

Hansteen, V. H. 1993, ApJ, 402, 741

He, J.-S., Tu, C.-Y., \& Marsch, E. 2007, A\&A, 468, 307

Landi, E., Del Zanna, G., Young, P. R., et al. 2006, ApJS, 162, 261

Lemaire, P., Bocchialini, K., Aletti, V., Hassler, D., \& Wilhelm, K. 1999, Space Sci. Rev., 87, 249

Mariska, J. T. 1992, The Solar Transition Region (Cambridge: Cambridge Univ. Press)

Marsch, E., Wiegelmann, T., \& Xia, L.-D. 2004, A\&A, 428, 629

Marsch, E., Zhou, G.-Q., He, J.-S., \& Tu, C.-Y. 2006, A\&A, 457, 699

Peter, H. 2001, A\&A, 374, 1108

Peter, H., \& Judge, P. G. 1999, ApJ, 522, 1148

Patsourakos, S., Vial, J.-C., Gabriel, A. H., \& Bellamine, N. 1999, ApJ, 522, 540

Raju, K. P., Srikanth, R., \& Singh, J. 1998, Sol. Phys., 178, 251

Ravindra, B., \& Venkatakrishnan, P. 2003, Sol. Phys., 215, 239

Reeves, E. M. 1976, Sol. Phys., 46, 53

Seehafer, N. 1978, Sol. Phys., 58, 215

Simon, G. W., \& Leighton, R. B. 1964, ApJ, 140, 1120

Schrijver, C. J. 1997, in The Corona and the Solar Wind Near Minimum Activity, ed. A. Wilson, ESA SP-404 (Noordwijk: ESA), 149

Teriaca, L., Banerjee, D., \& Doyle, J. G. 1999, A\&A, 349, 636

Teriaca, L., Madjarska, M. S., \& Doyle, J. G. 2002, A\&A, 392, 309

Tian, H., Tu, C.-Y., He, J.-S., \& Marsch, E. 2007, Adv. Space Res., 39, 1853

Tian, H., Tu, C.-Y., Marsch, E., He, J.-S., \& Zhou, G.-Q. 2008, A\&A, 478, 915

Tu, C.-Y., Zhou, C., Marsch, E., et al. 2005a, Science, 308, 519

Tu, C.-Y., Zhou, C., Marsch, E., et al. 2005b, ApJ, 624, L133

Wiegelmann, T., \& Solanki, S. K. 2004, Sol. Phys., 225,227

Wiegelmann, T., Xia, L.-D., \& Marsch, E. 2005, A\&A, 432, L1

Wilhelm, K., Lemaire, P., Dammasch, I. E., et al. 1998, A\&A, 334,685

Wilhelm, K., Marsch, E., Dwivedi, B. N., \& Feldman, U. 2007, Space Sci. Rev., in press

Xia, L.-D. 2003, Ph.D. Thesis, Göttingen: Georg-August-Univ

Xia, L.-D., Marsch, E., \& Wilhelm, K. 2004, A\&A, 424, 1025 